\title{
Taux de maladies chroniques au Canada : quel dénominateur utiliser pour l'estimation de l'ensemble de la population?
}

\author{
J. Ellison, M.P.H. (1); C. Nagamuthu, M.P.H. (2); S. Vanderloo, M. Sc. (3); B. McRae (1); C. Waters, B. Sc. (1)
}

Cet article a fait l'objet d'une évaluation par les pairs.

\section{Résumé}

Introduction : Les taux de maladies chroniques du Système canadien de surveillance des maladies chroniques (SCSMC) de l'Agence de la santé publique du Canada sont fondés sur les données administratives sur la santé fournies par les ministères de la Santé des provinces et des territoires. Les dénominateurs utilisés pour calculer ces taux reposent sur des estimations de population tirées des dossiers d'assurance-maladie, données toutefois pas systématiquement accessibles à tous les chercheurs. Le recensement de Statistique Canada constitue quant à lui une autre source d'estimation de la taille de la population. Notre étude visait d'abord à calculer les principaux écarts entre les dénominateurs de population à partir des estimations du SCSMC et à partir de celles de Statistique Canada et ensuite à déterminer les causes à l’origine des écarts entre ces sources de données.

Méthodologie : Nous avons comparé pour 2009 les dénominateurs fournis par le SCSMC et ceux fournis par Statistique Canada. Le dénominateur du SCSMC a été ajusté pour tenir compte des composantes de la croissance (naissances, décès, émigrants et immigrants) tirées des données de recensement de Statistique Canada.

Résultats : Le dénominateur non ajusté du SCSMC était de 34429804 personnes, soit une différence de $+3,2 \%$ par rapport à l'estimation de population de Statistique Canada pour 2009. Après ajustement du dénominateur du SCSMC pour tenir compte des composantes de la croissance, la différence entre les deux estimations s'est trouvé réduite à 431323 personnes, soit un écart de 1,3\% . L'estimation tirée du SCSMC constitue une surestimation par rapport à celle de Statistique Canada. Le plus grand écart entre les deux estimations relève de la composante de croissance liée à l'immigration, alors que l'écart le moindre provient de la composante de croissance liée à la population émigrante.

Conclusions : Disposant des descriptions de données par source de données, les chercheurs peuvent choisir quelle estimation de la population utiliser dans leurs calculs des fréquences de maladies.

Mots-clés : Système canadien de surveillance des maladies chroniques, dénominateur, recensement, estimations de la population au Canada, surveillance des maladies, mesures de la fréquence de maladies, données administratives sur la santé

\section{Introduction}

De nombreux pays, dont le Canada, l'Australie, la France et l'Italie, sont dotés de bases de données administratives sur la santé mises en œuvre ou prises en charge par des gouvernements qui fournissent un régime universel de soins médicaux ${ }^{1}$. Ces données administratives sur la santé sont recueillies par l'administration des services de soins de santé à intervalles réguliers ${ }^{2}$. Elles sont utilisables pour planifier les services de santé,

\section{Points saillants}

- Il importe d'utiliser des estimations de la population précises pour mesurer le fardeau des maladies chroniques.

- Les auteurs ont calculé les principaux écarts entre les dénominateurs de la population fournis par le Système canadien de surveillance des maladies chroniques et par Statistique Canada et ont cherché les causes les plus plausibles pour expliquer ces écarts.

- Le dénominateur non ajusté du SCSMC était de 34429804 personnes, soit de $3,2 \%$ supérieur à l'estimation de la population fournie par Statistique Canada pour 2009. Après ajustement de ce dénominateur pour tenir compte des composantes de la croissance, la différence entre les deux estimations a été réduite à 431323 personnes, soit un écart de 1,3\%.

- Le plus grand écart entre les deux estimations relevait de la composante de croissance liée à la population immigrante et l'écart le moindre de la composante de croissance liée à la population émigrante.

faire état des évaluations de rendement, éclairer la prise de décisions cliniques et répondre à des questions en matière de recherche $^{2}$. Elles servent aussi pour la surveillance des maladies ${ }^{3-7}$. Des mesures de fréquence des maladies, comme les taux de prévalence, d'incidence et de mortalité, contribuent à décrire le fardeau des maladies au sein d'une population. Grâce à cette information, les spécialistes des politiques, de l'économie de la santé et de la santé publique peuvent prendre des décisions éclairées. Il est

Rattachement des auteurs :

1. Centre de prévention des maladies chroniques, Agence de la santé publique du Canada, Ottawa (Ontario), Canada

2. Département des sciences de la santé publique, École de santé publique, Université de l'Alberta, Edmonton (Alberta), Canada

3. Programme d'épidémiologie clinique, Institut de recherche de l'Hôpital d'Ottawa, Ottawa (Ontario), Canada

Correspondance : Joellyn Ellison, Centre de prévention des maladies chroniques, Agence de la santé publique du Canada, 785, avenue Carling, $6^{\mathrm{e}}$ étage, 623 A-3, Ottawa (Ontario) K1A 0K9; tél. : 613-797-8721; téléc. : 613-941-2057; courriel : joellyn.ellison@phac-aspc.gc.ca 
donc essentiel que les chercheurs utilisent des dénominateurs appropriés pour le calcul de ces mesures, car les taux d'incidence, de prévalence et de mortalité se calculant à partir d'un numérateur et d'un dénominateur, une sélection inadéquate de la population totale peut produire des estimations biaisées des taux de survenue de maladies et de décès ${ }^{8}$. Bien souvent, ces estimations de population constituent les sources les plus pertinentes pour estimer le nombre de personnes à risque pour une maladie.

L'une des sources pour les estimations de population associées au dénominateur est Statistique Canada. Les données sur les citoyens (incluant les résidents permanents) et les résidents non permanents et leurs familles vivant au Canada sont recueillies par Statistique Canada dans le cadre de son recensement quinquennal ${ }^{9}$. La majeure partie de la population remplit elle-même un questionnaire de recensement et le renvoie par la poste ou par voie électronique?.

Le recensement a pour objectif de fournir de l'information sur les caractéristiques individuelles et sociales de la population canadienne ${ }^{9}$. Les estimations sont calculées en fonction de ces données de recensement et ajustées pour tenir compte du sousdénombrement ou du surdénombrement ${ }^{10}$.

Le Système canadien de surveillance des maladies chroniques (SCSMC) de l'Agence de la santé publique du Canada (ASPC) constitue une autre source pour les estimations de population associées au dénominateur. Il s'agit d'un réseau de collaboration entre les systèmes de surveillance des maladies chroniques aux échelles fédérale, provinciale et territoriale, soutenu par l'ASPC. Les estimations du dénominateur reposent sur le nombre de personnes détenant une police d'assurance-maladie valide à un moment quelconque au cours de l'exercice financier. Le SCSMC permet de déterminer le nombre de Canadiens atteints d'une maladie chronique, grâce aux interactions avec le système de soins de santé et en fonction des codes de diagnostics et de procédures, information qui s'ajoute au large éventail de données sur le fardeau des maladies au Canada. Ce système intègre des données globales sur le diabète, l'hypertension, la cardiopathie ischémique, l'infarctus aigu du myocarde, l'insuffisance cardiaque, la maladie mentale, l'ostéoporose, l'asthme, la maladie pulmonaire chronique, la sclérose en plaques et le parkinsonisme.
Cette étude est la première à comparer à l'échelle nationale le dénominateur du SCSMC, établi à partir des données administratives sur la santé, et les estimations de Statistique Canada sur la population. Il existe à l'échelle provinciale une étude du ministère de la Santé et du Bien-être de l'Alberta comparant les données du registre du Régime d'assurance-maladie de l'Alberta (AHCIP) et celles du recensement de $2006^{11}$. Cette étude a révélé un sousdénombrement de 0,0988 \% (3249 / 3287 101) en lien avec les données du registre de l'AHCIP de 2006-2007 par rapport à celles de Statistique Canada. Cependant, en juin 2015, l'estimation de l'AHCIP était plus élevée que celle de Statistique Canada ${ }^{12}$. L'ASPC a réalisé aussi une analyse similaire en 2012, dans le cadre de laquelle elle mettait en parallèle le dénominateur du SCSMC (exercice financier 2006-2007) et l'estimation de la population fournie par Statistique Canada (recensement de 2006), révélant une surestimation d'environ 3,9 \% (955358 / 24258902) pour le dénominateur du SCSMC. Cet écart était toutefois difficile à interpréter, puisque les données du SCSMC pour le Québec et Terre-Neuve-et-Labrador avaient été exclues en raison de leur mauvaise qualité et que les estimations de Statistique Canada avaient été utilisées à leur place. Au Québec, l'Institut national de santé publique du Québec (INSPQ) n'avait pas été en mesure de fournir de données sur les Canadiens ne souffrant pas de maladie. Quant à TerreNeuve-et-Labrador, les cartes d'assurancemaladie antérieures à 2008 ne comportant pas de date d'expiration, la base de données du régime de soins médicaux comptait des doublons.

Notre étude visait à la fois à calculer les principaux écarts entre les estimations de population issues du SCSMC et celles issues de Statistique Canada et à déterminer les causes à l'origine de ces écarts. Notre objectif était que les résultats de cette étude permettent d'orienter les décisions des chercheurs et des analystes dans leur choix de l'estimation de population associée au dénominateur pour le calcul des fréquences des maladies.

\section{Méthodologie}

\section{Sources de données}

Le SCSMC utilise des bases de données administratives provinciales et territoriales pour assurer la surveillance des maladies chroniques chez les Canadiens ${ }^{4-7}$. Ce système a été développé grâce au jumelage de trois bases de données administratives et d'identifiants uniques valides : (1) le fichier du registre d'assurance-maladie, (2) le dossier des services médicaux rémunérés à l'acte et certains dossiers de services médicaux soumis au système de facturation fictive et (3) des dossiers hospitaliers rendant compte, par le biais de codes de diagnostics et de procédures, des interactions liées aux soins de courte durée en milieu hospitalier ${ }^{7}$. Ces bases de données sur la santé consignent les interactions liées aux soins de santé administrés à des résidents admissibles aux services de soins de santé provinciaux ou territoriaux ${ }^{7}$. Le fichier du registre d'assurance-maladie renferme des renseignements personnels, dont un identifiant unique valide à vie, permettant de jumeler ces trois bases de données ${ }^{13}$.

Ce registre d'assurance-maladie contient aussi un dossier pour chacun des résidents en vie et admissible aux soins de santé à un moment quelconque au cours de l'exercice financier. Les personnes décédées sont donc consignées dans l'exercice au cours duquel elles sont décédées. Par ailleurs, ce sont les programmes de financement du gouvernement fédéral qui assurent à certains sous-groupes de la population (Premières Nations et Inuits, demandeurs d'asile, anciens combattants admissibles, détenus de pénitenciers fédéraux et militaires actifs des Forces canadiennes et de la GRC [Gendarmerie royale du Canada]) la prestation de services et d'avantages non assurés par les gouvernements provinciaux et territoriaux, notamment la couverture de soins dentaires et de la vue ainsi que la couverture de fournitures médicales et de certains médicaments ${ }^{14,15}$. Les Premières Nations et les Inuits bénéficient aussi d'un régime provincial d'assurance-maladie, et leurs données tirées du fichier de registre d'assurance-maladie ont donc été saisies par le SCSMC.

Les membres des Forces canadiennes et de la GRC ainsi que les détenus de pénitenciers fédéraux ${ }^{15}$ ne sont en principe pas saisis par le SCSMC (environ 110000 personnes par année). Les chiffres ayant servi à calculer le dénominateur du SCSMC proviennent des données transmises à l'ASPC par chacune des provinces et chacun des territoires, en date de novembre 2015, pour l'exercice 2009-2010. On a utilisé ces données car il s'agissait des données les plus récentes au moment de nos analyses. Elles concernaient les résidents 
couverts par un régime d'assurancemaladie valide à un moment quelconque au cours de l'exercice financier (incluant de ce fait les résidents décédés au cours de cet exercice) et étaient réparties en fonction de variables liées à la maladie et de caractéristiques individuelles comme l'âge et le sexe.

\section{Estimations de la population fournies par Statistique Canada}

Les estimations de la population fournies par Statistique Canada reposent sur les données de recensement. Bien que le but visé par Statistique Canada soit de dénombrer la population canadienne le jour du recensement en recueillant les données à un moment précis (perspective transversale de la population), il est inévitable qu'une certaine fraction de la population soit sous-dénombrée ou sur-dénombrée $(2,7 \%)(868657 / 32500000)^{16}$. Certaines personnes ne sont vraisemblablement pas comptabilisées, parce qu'elles ne sont pas au Canada au moment du recensement ou parce qu'elles vivent dans un logement collectif offrant des services de soins ou d'assistance, alors que d'autres personnes sont sans doute comptabilisées plusieurs fois (p. ex. étudiants vivant hors du domicile familial ayant rempli le questionnaire de recensement et figurant aussi sur celui de leurs parents $)^{16}$. Ces cas correspondent à ce qu'on définit comme un sousdénombrement et un sur-dénombrement ${ }^{17,18}$.

Statistique Canada mène des études d'évaluation postcensitaires au moyen d'un échantillon représentatif de la population pour calculer le nombre de personnes n'ayant pas été dénombrées ou ayant été dénombrées plusieurs fois au moment du recensement ${ }^{19}$. Les résultats de ces études sont combinés aux estimations du recensement pour fournir les estimations à jour de la population, qui reposent ainsi sur des estimations postcensitaires et intercensitaires $^{10}$. Les estimations intercensitaires correspondent aux estimations de population réalisées entre deux recensements ${ }^{10}$. Ces ajustements permettent d'obtenir des estimations presque exhaustives des effectifs de population.

\section{Cohortes d'étude}

Nous avons comparé, par groupe d'âge, le dénominateur du SCSMC pour l'exercice 2009-2010 et l'estimation de population de Statistique Canada pour 2009²0. Les données ont été recueillies selon l'âge au 31 mars pour le dénominateur du SCSMC et au $1^{\text {er }}$ juillet pour Statistique Canada. Le dénominateur du SCSMC a été ajusté pour tenir compte des composantes de la croissance (naissances, décès, émigrants et immigrants) tirées des fichiers de données de recensement de Statistique Canada (tableau 1) ${ }^{21}$. Les données utilisées pour le dénominateur du SCSMC incluaient la migration interprovinciale (les résidents ayant déménagé d'une province ou un territoire à un autre ont été de ce fait comptés plus d'une fois) alors que les estimations de Statistique Canada avaient été ajustées pour tenir compte de la migration interprovinciale, de l'immigration nette et des décès ${ }^{26}$.

Nous avons mené des analyses à l'aide du logiciel SAS Enterprise Guide, version 4.1 (SAS Institute Inc., Cary [Caroline du Nord], États-Unis). Pour illustrer les écarts entre les estimations de population du SCSMC et celles de Statistique Canada, nous avons réalisé nos analyses avec ajustement et sans ajustement du dénominateur du SCSMC en fonction des composantes de la croissance. Le tableau 2 offre une synthèse des définitions de données par source.

\section{Méthodes statistiques}

L'écart global en pourcentage entre le dénominateur du SCSMC et l'estimation de population déterminée par Statistique
Canada a été calculé ainsi, pour le Canada et par province/territoire :

[(dénominateur du SCSMC - estimation de population de Statistique Canada) / estimation de population de Statistique Canada] $\times 100$.

Pour examiner l'incidence sur les taux la plus forte, dans tous les groupes d'âge, en fonction des deux sources de données, nous avons comparé les taux de prévalence, d'incidence et de mortalité du diabète en utilisant le dénominateur du SCSMC et en utilisant l'estimation de population de Statistique Canada. Pour recenser les décès toutes causes confondues à l'échelle provinciale et territoriale, nous avons utilisé les données du SCSMC. Nous avons calculé les taux en utilisant les estimations de population pour le dénominateur tirées des deux sources de données :

- Prévalence du diabète $=$ [Nombre total de personnes pour lesquelles le cas a été déterminé au cours de la période de collecte de données ou cas prévalents / Titulaires d'une couverture d'assurancemaladie valide au cours de la période de collecte de données] $\times 100$ ou Prévalence du diabète $=[$ Nombre total de personnes pour lesquelles le cas a été déterminé au cours de la période de collecte de données ou cas prévalents / Estimation de la population par Statistique Canada] $\times 100$
TABLEAU 1

Dénominateur du SCSMC ajusté en fonction des méthodes utilisées par Statistique Canada ${ }^{22}$, Canada, 2009

\begin{tabular}{|lcc|}
\hline \multicolumn{1}{|c|}{ Composante de la croissance } & Ajustement & Ampleur du résultat ajusté \\
\hline Naissances $^{23}$ & $379373 / 3$ & 126457 \\
\hline Décès $^{23}$ & $237138 / 3$ & 79046 \\
\hline Immigrants $^{24}$ & $270581 / 3$ & 90193 \\
\hline Émigrants $^{24}$ & $52335 / 3$ & 17445 \\
\hline $\begin{array}{l}\text { Solde des résidents non } \\
\text { permanents }\end{array}$ & 34531 & 34531 \\
\hline & Hors pays : $\left(259234^{*} 1,5\right)-$ & 302440 \\
\hline
\end{tabular}

Abréviation : SCSMC, Système canadien de surveillance des maladies chroniques.

Remarques : Dénominateur du SCSMC ajusté $(n=33779692)$ = Dénombrement SCSMC ( $n=34429804)$ - naissances ( $n=126457)$ - décès $(n=79046)$ - immigrants $(n=90193)$ - émigrants $(n=17445)$ - solde des résidents non permanents $(n=34531)$ - migrants au pays et hors pays $(n=302440)$.

Après l'ajustement pour le SCSMC, la différence entre les deux dénominateurs est de 431296 (1,3%). 
TABLEAU 2

Descriptions des données du dénominateur par source (Système canadien de surveillance des maladies chroniques et Statistique Canada), 2009

\begin{tabular}{|c|c|c|}
\hline $\begin{array}{l}\text { Sources de } \\
\text { données }\end{array}$ & $\begin{array}{l}\text { Dénominateur du SCSMC de } \\
\text { I'ASPC }\end{array}$ & Estimation de population de Statistique Canada \\
\hline $\begin{array}{l}\text { Type } \\
\text { d'estimation }\end{array}$ & $\begin{array}{l}\text { Estimation fondée sur une période } \\
\text { (Canadiens détenant une police } \\
\text { d'assurance-maladie valide au cours } \\
\text { de l'exercice financier) }\end{array}$ & $\begin{array}{l}\text { Estimation ponctuelle (estimation du nombre de } \\
\text { Canadiens recensés) }\end{array}$ \\
\hline Inclusions & $\begin{array}{l}\text { 1) Décès survenant pendant l'exercice } \\
\text { financier } \\
\text { 2) Canadiens migrant à l'intérieur du } \\
\text { Canada pendant l'exercice financier } \\
\text { (double comptage) }\end{array}$ & $\begin{array}{l}\text { 1) Échantillons représentatifs des Canadiens } \\
\text { 2) Ajustements selon les composantes de la } \\
\text { croissance (naissances, décès, émigrants et } \\
\text { immigrants) et les personnes non recensées (hors } \\
\text { du pays pendant le recensement) ou dénombrées } \\
\text { deux fois (étudiants ne vivant plus au domicile } \\
\text { familial) }\end{array}$ \\
\hline Exclusions & $\begin{array}{l}\text { Canadiens couverts par une } \\
\text { assurance du gouvernement fédéral }\end{array}$ & $\begin{array}{l}\text { Canadiens hors pays pendant le recensement ou } \\
\text { vivant dans un logement collectif, mais pour } \\
\text { lesquels un ajustement a été effectué }\end{array}$ \\
\hline $\begin{array}{l}\text { Utilisation/ } \\
\text { rôle }\end{array}$ & $\begin{array}{l}\text { Utilisé conjointement avec le } \\
\text { numérateur du SCSMC (les Canadiens } \\
\text { exposés à un trouble de santé sont } \\
\text { inclus dans le dénominateur) }\end{array}$ & $\begin{array}{l}\text { Utilisé conjointement avec le numérateur du } \\
\text { SCSMC (inclut les Canadiens exposés à un trouble } \\
\text { de santé) }\end{array}$ \\
\hline
\end{tabular}

Abréviations : ASPC, Agence de la santé publique du Canada; SCSMC, Système canadien de surveillance des maladies chroniques.

- Incidence du diabète $=[$ Nombre total de cas incidents / (Nombre total de titulaires d'une couverture d'assurancemaladie valide au cours de la période de collecte de données - Cas prévalents au début de l'exercice) $] \times 1000$ ou Incidence du diabète $=[$ Nombre total de cas incidents / (Estimation de la population par Statistique Canada - Cas prévalents au début de l'exercice) $] \times 1000$

- Mortalité toutes causes confondues = [Nombre total de décès recensés par le SCSMC / Population totale recensée par le SCSMC] $\times 100000$ personnes ou Mortalité toutes causes confondues $=$ [Nombre total de décès recensés par le SCSMC / Estimation de la population par Statistique Canada] $\times 100000$ personnes.

Ces taux ont été calculés à l'aide de macros SAS (codes préprogrammés) et les comptes ont été arrondis aléatoirement ${ }^{26}$. Les données de mortalité utilisées dans l'estimation de population de Statistique Canada provenaient des fichiers de statistiques de l'état civil de 2009 (pour assurer leur concordance avec ceux du SCSMC pour l'exercice 2009-201027. Pour évaluer l'incidence des taux de mortalité sur le calcul de l'espérance de vie au moyen des deux sources de dénominateurs, nous avons comparé, par sexe et par groupe d'âge, l'espérance de vie calculée au moyen du dénominateur du SCSMC pour chacune des maladies suivies par le SCSMC avec l'espérance de vie calculée au moyen de l'estimation de population de Statistique Canada. La stratification des données du SCSMC a été faite par sexe et selon les 18 groupes d'âge standard ( 1 à 4 ans, 5 à 9 ans, ..., 80 à 84 ans, 85 ans et plus) puis nous avons créé une table de survie pour le nombre de personnes ayant reçu un code de diagnostic lié à une maladie et pour le nombre de personnes n'ayant reçu aucun code de diagnostic. La fonction de Gompertz a servi à obtenir une estimation précise de l'espérance de vie au dernier intervalle d'âge ouvert ( 85 ans et plus) de façon à s'approcher de la table de survie ${ }^{28,29}$.

Comme la situation vis-à-vis de la maladie n'était pas connue chez les enfants de moins d'un an, nous avons utilisé les taux de mortalité pour la population canadienne selon le sexe de 2004 à 2006 de Statistique Canada pour modéliser la mortalité des individus atteints de maladie et celle des individus non atteints de maladie. Les taux de mortalité selon le sexe des enfants de la naissance à 1 an utilisés pour construire la table de survie correspondaient à ceux des nourrissons non atteints de maladie, car les taux de mortalité des nourrissons atteints de la maladie n'étaient pas disponibles. Le groupe d'âge des enfants de la naissance à 1 an affichant un taux de mortalité élevé, nous avons présumé que le nombre de nourrissons atteints de maladie et celui de nourrissons non atteints étaient approximativement les mêmes.

\section{Résultats}

Nos résultats révèlent que le dénominateur du SCSMC et les estimations de la population produites par Statistique Canada diffèrent. Plus précisément, on observe une surestimation de la population dans le SCSMC par rapport à l'estimation globale fournie par Statistique Canada.

Le plus grand écart entre les deux estimations relève de la composante de croissance liée à la population immigrante (302 440), l'écart le moindre, de la composante de croissance liée à la population émigrante (17 445) (tableau 1). L'inclusion des décès dans le calcul du dénominateur du SCSMC a conduit à une différence de 1081408 personnes entre le dénominateur du SCSMC (34 429 804) et l'estimation de population de Statistique Canada (33 348 396), soit un écart de + 3,2 \% (1 081 408/33 348 396) (tableau 3).

Après ajustement du dénominateur du SCSMC pour tenir compte des composantes de la croissance, nous avons observé une différence de 431296 personnes entre le dénominateur du SCSMC (33 779 692) et l'estimation de population de Statistique Canada (33 348 396), soit un écart de + 1,3 \% (431 296/33 348 396) (tableau 1). Le plus grand écart observé était dans le groupe des 85 ans et plus $(+20,5 \%$; $126453 / 617$ 160) et le moindre chez les 20 à 24 ans (- 0,8 \%; 17 757/2 322 497) (tableau 3$)$.

Par province ou territoire, l'écart le plus important a été observé dans les Territoires du Nord-Ouest (+ 13,0 \%; 5 600/42 965), et le moindre au Québec (+ 0,5\%; 37 595/7 737 335) (tableau 4). Nous avons constaté une tendance similaire lorsque les décès étaient exclus du dénominateur du SCSMC. Toutefois, après ajustement en fonction des composantes de la croissance, l'écart s'est trouvé réduit à 431296 personnes ( $\Delta 1,3 \%$; 431 296/33 348 396) (tableau 1).

Pour l'ensemble des Canadiens, le taux de prévalence du diabète calculé avec le 
TABLEAU 3

Estimations de la population par groupe d'âge, Canada, 2009

\begin{tabular}{|c|c|c|c|}
\hline $\begin{array}{l}\text { Groupes d'âge } \\
\text { (ans) }\end{array}$ & $\begin{array}{l}\text { Dénominateur du } \\
\text { SCSMC }^{\mathrm{a}}\end{array}$ & $\begin{array}{c}\text { Estimation de } \\
\text { population de } \\
\text { Statistique Canadab }\end{array}$ & $\begin{array}{l}\text { Pourcentage de l'écart } \\
(\%)\end{array}$ \\
\hline 1 à 4 & 1540368 & 1464423 & 5,2 \\
\hline 5 à 9 & 1845877 & 1798812 & 2,6 \\
\hline 10 à 14 & 2009792 & 1972894 & 1,9 \\
\hline 15 à 19 & 2264299 & 2250692 & 0,6 \\
\hline 20 à 24 & 2304740 & 2322497 & $-0,8$ \\
\hline 25 à 29 & 2358661 & 2348492 & 0,4 \\
\hline 30 à 34 & 2310455 & 2258092 & 2,3 \\
\hline 35 à 39 & 2377282 & 2297458 & 3,5 \\
\hline 40 à 44 & 2512797 & 2480011 & 1,3 \\
\hline 45 à 49 & 2858523 & 2787129 & 2,6 \\
\hline 50 à 54 & 2686876 & 2573413 & 4,4 \\
\hline 55 à 59 & 2322492 & 2215710 & 4,8 \\
\hline 60 à 64 & 1999094 & 1888212 & 5,9 \\
\hline 65 à 69 & 1480822 & 1406971 & 5,2 \\
\hline 70 à 74 & 1137703 & 1080535 & 5,3 \\
\hline 75 à 79 & 947527 & 909136 & 4,2 \\
\hline 80 à 84 & 728883 & 676759 & 7,7 \\
\hline 85 et plus & 743613 & 617160 & 20,5 \\
\hline Tous âges & 34429804 & 33348396 & 3,2 \\
\hline
\end{tabular}

${ }^{a}$ Les données ayant permis de déterminer le dénominateur du SCSMC ont été fournies par les provinces et les territoires à compter de novembre 2015 (v2014).

${ }^{\mathrm{b}}$ Estimations postcensitaires de la population par Statistique Canada (Recensement de 2006) ${ }^{20}$.

TABLEAU 4

Estimations de la population par province ou territoire, Canada, 2009

\begin{tabular}{|lrrr}
\hline \multicolumn{1}{|c}{ Province ou territoire } & $\begin{array}{c}\text { Dénominateur } \\
\text { du SCSMC }\end{array}$ & $\begin{array}{r}\text { Estimations de la population de } \\
\text { Statistique Canada }\end{array}$ & $\begin{array}{c}\text { Pourcentage de } \\
\text { l'écart (\%) }\end{array}$ \\
\hline Terre-Neuve-et-Labrador & 537862 & 504141 & 6,7 \\
\hline île-du-Prince-Édouard & 148911 & 139593 & 6,7 \\
\hline Nouvelle-Écosse & 989707 & 931622 & 6,2 \\
\hline Nouveau-Brunswick & 755910 & 742506 & 1,8 \\
\hline Québec & 7774930 & 7737335 & 0,5 \\
\hline Ontario & 13563855 & 12928815 & 4,9 \\
\hline Manitoba & 1239544 & 1204232 & 2,9 \\
\hline Saskatchewan & 1067733 & 1015590 & 5,1 \\
\hline Alberta & 3711026 & 3621681 & 2,5 \\
\hline Colombie-Britannique & 4524374 & 4415160 & 2,5 \\
\hline Yukon & 33745 & 33342 & 1,2 \\
\hline Territoires du Nord-Ouest & 48565 & 42965 & 13,0 \\
\hline Nunavut & 33642 & 31414 & 7,1 \\
\hline Canada & 34429804 & 33348396 & 3,2 \\
\hline
\end{tabular}

${ }^{a}$ Les données ayant permis de déterminer le dénominateur du SCSMC ont été fournies par les provinces et les territoires à compter de novembre 2015 (v2014).

${ }^{\mathrm{b}}$ Estimations postcensitaires de la population par Statistique Canada (Recensement de 2006) ${ }^{20}$. dénominateur du SCSMC $(7,2 \%$; $2489520 / 34429804)$ était de 4,00 \% $(-0,3 / 7,5)$ inférieur à celui calculé avec l'estimation de population de Statistique Canada $(7,5 \% ; 2489$ 520/33 348 396), alors que le taux d'incidence calculé avec le dénominateur du SCSMC $(6,4$ pour $1000 ; 218$ 240/34 211564 ) était de 3,03\% $(-0,2 / 6,6)$ inférieur à celui calculé avec l'estimation de population de Statistique Canada $(6,6$ pour $1000 ; 218240 / 33130156)$. Le taux de mortalité total toutes causes confondues calculé avec le dénominateur du SCSMC (669,2 pour $100000 ; 230408 / 34429804)$ était de $3,1 \%(-21,7 / 690,9)$ inférieur à celui calculé avec l'estimation de population de Statistique Canada (690,9 pour 100000; 230408/33348396; tableau 5). L'espérance de vie à la naissance calculée avec le dénominateur du SCSMC s'établissait à 82,9 ans, et celle calculée avec l'estimation de population de Statistique Canada à 81,2 ans.

\section{Analyse}

L'une des différences entre le dénominateur du SCSMC et l'estimation de population de Statistique Canada est que cette dernière fournit une perspective transversale de la population à une date donnée (date du recensement) alors que le dénominateur du SCSMC fournit une estimation de la population exposée (ou " à risque ») sur une année. Bien que les taux de prévalence et d'incidence puissent être calculés pour une date précise, ils sont plus couramment calculés pour une période. Les chercheurs doivent prendre note que des dénominateurs différents sont susceptibles de produire des taux de prévalence, d'incidence et de mortalité et un calcul de l'espérance de vie différents. Par exemple, un militaire qui consulte un médecin à l'hôpital pour son diabète ne sera pas enregistré dans le SCSMC car les militaires forment un sous-groupe de population couvert par une assurance fédérale. Les estimations de Statistique Canada sont donc les plus appropriées pour le dénominateur entrant dans le calcul de fréquence d'une maladie. Il convient toutefois de noter que le dénominateur du SCSMC est le plus approprié pour l'estimation de fréquence d'une maladie lorsque des données administratives sont utilisées (c'est-à-dire lorsque le numérateur repose sur les données du SCSMC).

Par ailleurs, un chercheur pourrait souhaiter calculer la prévalence du diabète en 2006 pour l'ensemble des Canadiens. Il 
TABLEAU 5

Taux de prévalence, d'incidence et de mortalitéa du diabète par population, Canada, 2009

\begin{tabular}{lccc}
\hline Statistique & $\begin{array}{c}\text { Dénominateur du } \\
\text { SCSMC }^{\mathrm{b}}\end{array}$ & $\begin{array}{c}\text { Estimations de } \\
\text { population de } \\
\text { Statistique Canada }\end{array}$ & $\begin{array}{c}\text { Pourcentage de l'écart } \\
(\%)\end{array}$ \\
\hline Prévalence & 7,2 & 7,5 & $-4,0$ \\
\hline Incidence & 6,4 pour 1000 & 6,6 pour 1000 & $-3,0$ \\
\hline & Total (excluant les décès au sein de la population) & \\
\hline Mortalité & 669,2 pour 100000 & 690,9 pour 100000 & $-3,1$ \\
\hline
\end{tabular}

Remarques : Cette étude a pu être réalisée grâce à la collaboration de l'Agence de la santé publique du Canada (ASPC), des gouvernements provinciaux, soit la Colombie-Britannique, l'Alberta, la Saskatchewan, le Manitoba, l'Ontario, le Québec, le Nouveau-Brunswick, l'île-du-Prince-Édouard, la Nouvelle-Écosse et Terre-Neuve-et-Labrador, et des gouvernements des territoires, soit le Yukon, les Territoires du Nord-Ouest et le Nunavut. Les opinions, les résultats et les conclusions présentés dans cette étude n'engagent que les auteurs; ils ne sont nullement sanctionnés par la Colombie-Britannique, la Saskatchewan, le Manitoba, l'Ontario, le Québec, le Nouveau-Brunswick, l'île-du-Prince-Édouard, la Nouvelle-Écosse, Terre-Neuve-et-Labrador, le Yukon, les Territoires du Nord-Ouest et le Nunavut et ne devraient pas être perçus comme tels.

${ }^{a}$ Les comptes du SCSMC ont été arrondis aléatoirement.

b Les données ayant permis de déterminer le dénominateur du SCSMC ont été fournies par les provinces et les territoires à compter de novembre 2015 (v2014).

' Estimations postcensitaires de la population par Statistique Canada (Recensement de 2006) ${ }^{20}$.

pourrait alors utiliser le nombre de cas de diabète recensés en 2006 dans le SCSMC et décider d'utiliser les estimations de Statistique Canada comme dénominateur (ce qui correspond à la population canadienne totale en 2006). Cependant, le risque d'être atteinte de la maladie utilisé dans le numérateur pourrait ne pas cadrer avec les estimations de Statistique Canada (pourrait ne pas être représentatif de cette population). Ou encore, une personne atteinte de diabète pourrait être décédée avant la date de recensement de 2006 mais avoir été recensée dans la base de données avant son décès. Dans ce scénario, la personne serait prise en compte dans le calcul du numérateur mais pas dans celui du dénominateur, produisant ainsi une estimation inexacte de la prévalence du diabète au Canada en 2006.

Pour quantifier les lacunes liées aux données non incluses dans le dénominateur du SCSMC et dans l'estimation de population de Statistique Canada, une recherche plus approfondie est nécessaire, que ce soit sur la qualité des données tirées des registres d'assurance-maladie ou sur la quantification des sous-groupes de population (comme les émigrants) non inclus dans les deux sources de données.

Le SCSMC tient compte de presque toute la population canadienne lorsqu'il saisit des données du registre d'assurance-maladie. Les personnes qui déménagent dans une autre province ou un autre territoire au cours d'un exercice financier (migration interprovinciale/interterritoriale) et bénéficient de la protection d'une assurancemaladie valide sont comptées deux fois dans le calcul du dénominateur du SCSMC, pour une période de temps limitée.

L'écart entre le dénominateur ajusté du SCSMC et l'estimation de Statistique Canada (431296 personnes) peut être attribuable aux divergences dans les numéros valides et admissibles saisis dans les registres d'assurance-maladie, divergences susceptibles de découler de la fraude $^{30}$. De plus, les registres d'assurancemaladie peuvent comporter de l'information inexacte sur des décès, en raison des ressources et du temps requis pour traiter ce type d'information ${ }^{31}$. Statistique Canada a eu de la difficulté à recenser les Canadiens ayant émigré pour des motifs professionnels et qui sont sans domicile, mais on estime qu'ils sont peu nombreux $^{32-35}$. Les responsables des registres d'assurance-maladie comme ceux de Statistique Canada continuent à documenter ces données, à traiter de ces enjeux et à trouver des explications et des stratégies de compensation à ces phénomènes. On a également observé de légères différences d'âge (dans les groupes d'âge représentant les plus jeunes) qui pourraient s'expliquer par le fait que l'âge enregistré à des dates de référence précises pourrait différer dans les deux sources de données. La sous-estimation décelée par le gouvernement de l'Alberta ${ }^{11}$ pourrait être attribuable aux différentes méthodes utilisées par le Régime d'assurance-maladie de l'Alberta
(AHCIP) (comparaison des données du recensement de 2006) et la Division de la démographie de Statistique Canada (qui procède à l'ajustement du dénominateur du SCSMC propre à une province).

\section{Conclusion}

Nos résultats montrent l'importance de prendre une décision éclairée lors de la sélection des estimations de la population dans le cadre d'une recherche, car les estimations choisies ont une influence sur le calcul des taux. Nous avons constaté que même après l'ajustement du dénominateur du SCSMC pour tenir compte des décès et de la migration interprovinciale, celui-ci était supérieur à l'estimation de Statistique Canada.

Grâce à ces résultats, les chercheurs peuvent comparer les principales raisons expliquant les écarts entre le dénominateur du SCSMC et l'estimation de la population de Statistique Canada et ainsi choisir, dans le cadre de leurs projets, le dénominateur le plus approprié pour mesurer la fréquence de la maladie.

Nous sommes d'avis que le dénominateur du SCSMC est le plus représentatif dans le cas d'une population à risque cernée au moyen des données administratives sur la santé. Le dénominateur du SCSMC sert alors à mesurer la fréquence d'une maladie dans la mesure où il tient compte des personnes bénéficiant d'une protection d'assurancemaladie valide au cours de la période donnée. L'utilisation des estimations de population de Statistique Canada en guise de dénominateur conduit à une sousestimation de la taille de la population car le recensement est réalisé à un moment précis et les personnes absentes du Canada à ce moment alors qu'elles sont prises en compte dans le numérateur (prévalence). Même si l'ampleur des écarts dans les taux de diabète s'est révélée faible entre les deux sources, ces résultats pourraient avoir une petite incidence sur l'interprétation et les conclusions tirées d'études antérieures, dans le cadre desquelles la prévalence, l'incidence et la mortalité ont été évaluées au moyen des estimations de population de Statistique Canada pour le calcul du dénominateur de population.

\section{Remerciements}

Nous tenons à remercier les membres du Comité scientifique et technique du SCSMC. Cette étude a pu être réalisée grâce 
à la collaboration de l'ASPC, des gouvernements provinciaux (la ColombieBritannique, l'Alberta, la Saskatchewan, le Manitoba, l'Ontario, le Québec, le NouveauBrunswick, l'Île-du-Prince-Édouard, la Nouvelle-Écosse et Terre-Neuve-et-Labrador) et des gouvernements des territoires (le Yukon, les Territoires du Nord-Ouest et le Nunavut). Les interprétations, les résultats et les conclusions présentés dans cette étude n'engagent que les auteurs et ils ne sont nullement cautionnés par l'un des gouvernements susmentionnés. Ils ne devraient donc pas être perçus comme tels.

\section{Références}

1. Moise P. OECD ageing related disease study: using hospital administrative databases for a disease-based approach to studying health care systems. Paris : Organisation for Economic Co-operation and Development; 2001. Report IAG Y1-AG-8363 -01. PDF téléchargeable à partir du lien : http://www.oecd.org/sweden /1889879.pdf

2. Iron K, Manuel DG. Quality assessment of administrative data (QuAAD): an opportunity for enhancing Ontario's health data [Internet]. Toronto (Ont) : Institute for Clinical Evaluative Sciences; 2007. PDF téléchargeable à partir du lien: http://www.ices.on.ca/ /media/Files /Atlases-Reports/2007 / Quality -assessment-of-administrative-data /Full\%20report.ashx

3. Alaghehbandan R, MacDonald D. Use of administrative health databases and case definitions in surveillance of depressive disorders: a review. OA Epidemiol. 2013;1(3):1-7. doi: 10.13172 /2053-079x-1-1-539.

4. Robitaille C, Dai S, Waters C et collab. Diagnosed hypertension in Canada. JAMC. 2012;184(1):E49-56. doi: 10 $.1503 /$ cmaj. 101863 .

5. Robitaille C, Bancej C, Dai S et collab. Surveillance of ischemic heart disease should include physician billing claims: population-based evidence from administrative health data across seven Canadian provinces. BMC Cardiovasc Disord. 2013;13:88. doi: 10.1186/1471-2261-13-88.
6. Blais C, Dai S, Waters $C$ et collab. Assessing the burden of hospitalized and community-care heart failure in Canada. J Can Cardiol. 2014;30(3):352358. doi: 10.1016/j.cjca.2013.12.013.

7. Pelletier C, Dai S, Roberts KC, Bienek A, Onysko J, Pelletier L. Note de synthèse. Le diabète au Canada : perspective de santé publique sur les faits et chiffres. Maladies chroniques et blessures au Canada. 2012;33(1):61-62.

8. Fletcher RW, Fletcher SW. Clinical epidemiology: the essentials (4 éd). Baltimore (MD) : Lippincott, Williams and Wilkins; 2005. 251 p.

9. Statistique Canada. Recensement de la population [Internet]. Ottawa (Ont.) : Statistique Canada; 2012 [consulté le 22 juillet 2015]. Consultable à la page : http://www23.statcan.gc.ca/imdb/ p2SV_f.pl?Function = getSurvey\&SDDS $=3901 \&$ Item_Id $=114960$ \&lang $=$ en

10. Statistique Canada. Méthodes d'estimation de la population et des familles à Statistique Canada [Internet]. Ottawa (Ont.) : Statistique Canada; 2013 [consulté en juillet 2015]. Consultable à partir de la page : http://www.statcan.gc.ca/pub/91 -528-x/91-528-x2011001-fra.htm

11. Gouvernement de l'Alberta. Comparison of Alberta population counts between the AHCIP registry and the 2006 census. Edmonton (AB) : Alberta Health and Wellness; 2009. PDF téléchargeable à partir du lien : http:// www.health.alberta.ca/documents /Population-2006-Comparison-2009 .pdf

12. Alberta Health and Wellness. Health trends Alberta: weekly statistical report [Internet]. Edmonton (AB): Alberta Health and Wellness; 2015 [consulté le 22 décembre 2015]. Consultable à la page : http://www .health.alberta.ca/newsroom/health -trends.html

13. Santé Canada. Relever le défi posé par le diabète au Canada : premier rapport du Système national de surveillance du diabète (SNSD). Ottawa (Ont.) : Santé Canada; 2003. PDF téléchargeable à partir du lien : http://www.phac -aspc.gc.ca/ccdpc-cpcmc/ndss-snsd /francais/pubs_reports/pdf/NDSS_ French_Report_FINAL.pdf
14. Gouvernement du Canada. Services de santé non assurés pour les Premières Nations et les Inuits [Internet]. Ottawa (Ont.) : Gouvernement du Canada; [mis à jour le 1 septembre 2016; consulté le 22 juillet 2015]. Consultable à la page : http://canadiensensante.gc.ca /health-system-systeme-sante/services /non-insured-health-benefits-services -sante-non-assures/index-fra.php

15. Santé Canada. Rôle du fédéral dans la santé [Internet]. Ottawa (Ont.) : Santé Canada; [modifié le 7 octobre 2005; consulté le 22 juillet 2015]. Consultable à la page : http://canadiensensante .gc.ca/health-system-systeme-sante /cards-cartes/federal-role-fra.php\#a1

16. Statistique Canada. Estimation des erreurs de couverture de la population [Internet]. Ottawa (Ont.) : Statistique Canada; 2010 [consulté le 22 juillet 2015]. Consultable à la page : http://www12 .statcan.gc.ca/census-recensement /2006/ref/rp-guides/rp/coverage -couverture/cov-couv_p01-fra.cfm

17. Statistique Canada. Recensement de 2011 - Collecte [Internet]. Ottawa (Ont.) : Statistique Canada; [consulté le 22 juillet 2015]. Consultable à la page : http://www12.statcan.gc.ca /census-recensement/2011/ref/about -apropos/col-fra.cfm

18. Statistique Canada. Différences entre les comptes du recensement et les estimations démographiques de Statistique Canada [Internet]. Ottawa (Ont.) : Statistique Canada; [consulté le 22 juillet 2015]. Consultable à la page : http://www.statcan.gc.ca/fra /pa/estima

19. Statistique Canada. Rapport technique du Recensement de 2006 : Couverture [Internet]. Ottawa (Ont.) : Ministre de l'Industrie; 2010 [consulté le 22 juillet 2015]. Consultable à la page : http: / / ww w 12 . statcan .ca / census -recensement/2006/ref/rp-guides/rp /coverage-couverture/cov-couv_index -fra.cfm

20. Statistique Canada. Population par année, par province et territoire (Nombre) [Internet]. Ottawa (Ont.) : Statistique Canada; 2012 [consulté le 22 juillet 2015]. Consultable à la page : http://www.statcan.gc.ca/tables - tableaux/sum-som/102/cst01 /demo02a-fra.htm 
21. Statistique Canada. Estimation de la migration - guide de l'utilisateur [Internet]. Ottawa (Ont.) : Statistique Canada; 2011 [consulté le 22 juillet 2015]. Consultable à la page : http:// www23.statcan.gc.ca/imdb-bmdi /document/4101_D3_T1_V8-fra.htm

22. Statistique Canada. Méthodes d'estimation de la population et des familles à Statistique Canada. Ottawa (Ont.) : Statistique Canada; 2003. PDF téléchargeable à partir du lien : ht t p://publications.gc.ca/ C o l l e c t i o n - R / S t a t c a n / 91-528-XIF/0010391-528-XIF.pdf

23. Statistique Canada. Tableau 051-0013. Estimations des naissances, selon le sexe, Canada, provinces et territoires, annuel (personnes) [Internet]. Ottawa (Ont.) : Statistique Canada; [consulté le 9 juin 2015]. Consultable à la page : http:// www5.statcan.gc.ca/cansim/a26?id $=510013 \&$ retrLang $=$ fra\&lang $=$ fra

24. Statistique Canada. Tableau 051-0063. Composantes de l'accroissement démographique selon la division de recensement, le sexe et le groupe d'âge pour la période allant du $1^{\text {er }}$ juillet au 30 juin, basée sur la Classification géographique type (CGT) 2011, annuel (personnes) [Internet]. Ottawa (Ont.) : Statistique Canada; [consulté le 9 juin 2015]. Consultable à la page : http://www5 .statcan.gc.ca/cansim/a26? id = $510063 \&$ retrLang $=$ fra\&lang $=$ fra

25. Statistique Canada. Tableau 051-0012. Migrants interprovinciaux, selon le groupe d'âge et le sexe, Canada, provinces et territoires, annuel (personnes) [Internet]. Ottawa (Ont.) : Statistique Canada; [consulté le 9 juin 2015]. Consultable à la page : http://www5 .statcan.gc.ca/cansim/a26?id = 510012 \&retrLang $=$ fra\&lang $=$ fra

26. Statistique Canada. Estimations démographiques annuelles : Canada, provinces et territoires. Ottawa (Ont.) : Statistique Canada; 2012. PDF téléchargeable à partir du lien : http:// www.statcan.gc.ca/pub/91-215-x/91 -215-x2012000-fra.pdf
27. Statistique Canada. Tableau 051-0002. Estimations des décès, selon le sexe et le groupe d'âge, Canada, provinces et territoires; édition 2013 [Internet]. Ottawa (Ont.) : Statistique Canada; [consulté le 22 juillet 2015]. Consultable à la page : http://www5.statcan.gc.ca /cansim/a26?id $=0510002 \&$ pattern $=\&$ p2 $=31 \&$ stByVal $=1 \& \mathrm{p} 1=1 \&$ tabMode $=$ dataTable \&paSer $=\& \operatorname{csid}=\&$ retrLang $=$ fra\&lang $=$ fra

28. Chiang CL. The life table and its applications. Malabar (FL) : Robert E. Krieger Publishing Company; 1984. 336 p.

29. Hsieh JJ. A general theory of life construction and a precise abridged life table method. Biom J. 1991;33(2): 143-162.

30. Ministère de la Santé et des Soins de longue durée de l'Ontario (MSSO). Fraude à l'Assurance-santé [Internet]. Toronto (Ont.) : MSSO; 2009 [consulté le 22 décembre 2015]. Consultable à la page : http://www.health.gov.on.ca /fr/public/publications/ohip/card_ fraud.aspx

31. Iron K, Zagorski BM, Sykora K, Manuel DG. Living and dying in Ontario: an opportunity for improved health information. Toronto (Ont.) : Institute for Clinical Evaluative Sciences; 2008. Consultable à partir de la page : http:// www.ices.on.ca/Publications/Atlases - and-Reports / 2008/Living-and -dying-in-Ontario

32. DeVoretz DJ. Immigrant circulation and citizenship: Hotel Canada? Project Paper Series No. 09-04:1-19. [Internet]. Vancouver (C.-B.) : Fondation Asie Pacifique du Canada. PDF téléchargeable à partir du lien : http://www. asiapacific.ca/sites/default/files /filefield/PP_09_4_DD_HotelCanada .pdf

33. DeVoretz DJ. La province secrète du Canada : les 2,8 millions de Canadiens vivant à l'étranger. Project Paper Series No. 09-05 [Internet]. Vancouver (C.-B.) : Fondation Asie Pacifique du Canada; 2009. PDF téléchargeable à partir du lien : http://www.asiapacific .ca/sites/default/files/filefield /frOverseas_Canadians.pdf
34. Gaetz S, Gulliver T, Richter T. L'état de l'itinérance au Canada 2014. Toronto (Ont.) : 2014. PDF téléchargeable à partir du lien : http://rondpointdelitinerance .ca/sites/default/files/SOHC2014FR .pdf

35. Hwang SW. Homelessness and health. JAMC. 2001;164(2);229-233. 